Andrea Vannini Santesso Caiuby ${ }^{1}$, Paola Bruno de Araújo Andreoli², Sergio Baxter Andreoli ${ }^{3}$

1. Mestre, Pós-graduanda (Doutorado) do Departamento de Psiquiatria da Universidade Federal de São Paulo UNIFESP - São Paulo (SP), Brasil.

2. Doutora, Psicóloga da Prática Assistencial Multiprofissional do Hospital Israelita Albert Einstein HIAE - São Paulo (SP), Brasil.

3. Médico, Professor Afiliado do Departamento de Psiquiatria da Universidade Federal de São Paulo UNIFESP - São Paulo (SP), Brasil.

Submetido em 25 de Junho de 2009 Aceito em 22 de Fevereiro de 2010

Autor para correspondência: Andrea Vannini Santesso Caiuby Rua Dr. Bacelar, 368 - conjunto 142 Vila Clementino CEP: 04026-001 - São Paulo (SP), Brasil.

Fone: (11) 9224-7279

E-mail: andreacaiuby@hotmail.com

\section{Transtorno de estresse pós-traumático em pacientes de unidade de terapia intensiva}

\author{
Post-traumatic stress disorder in intensive care unit patients
}

\section{RESUMO}

O transtorno de estresse pós-traumático tem sido descrito em pacientes após tratamento em unidade de terapia intensiva. O objetivo foi revisar estudos sobre os aspectos psicológicos e as intervençóes terapêuticas destes pacientes após internação em unidade de terapia intensiva. Trinta e de transtorno de estresse pós-traumático variou de $17 \%$ a $30 \%$ e a incidência de $14 \%$ a $24 \%$. Os fatores de risco foram: história prévia de ansiedade, depressão ou pânico, ter memórias traumáticas ilusórias (memórias derivadas de formações psíquioito artigos foram incluídos. A prevalência cas como sonho e delirium), tempo de ventilação mecânica, experiências estressantes, crenças e comportamentos de característica depressiva. Doses altas de opióides, sintomas na retirada da sedação ou analgesia e o uso de lorazepam foram relacionados ao aumento de delirium e de memória ilusória. A sintomatologia do transtorno pode ser reduzida com a administraçáo de hidrocortisona, com a interrupção diária da sedação e não foram encontrados estudos de efetividade de intervenção psicológica.

Descritores: Transtornos de estresse pós-traumático/psicologia; Unidades de terapia intensiva; Adulto
$\mathrm{Na}$ última década, a compreensão de que as experiências emocionais de pacientes internados em unidade de terapia intensiva (UTI) podem ser estressantes e traumáticas têm levado os profissionais a investigar não somente os tratamentos curativo ou paliativo na UTI, mas também intervençóes para prevençáo de quadros emocionais prejudiciais à reabilitação global e à qualidade de vida do paciente após internação. ${ }^{(1-5)}$

Estudos demonstraram que a experiência de estar internado em UTI pode desencadear memórias traumáticas geradas pela vivência de ameaça de morte. ${ }^{(2-4,6)}$ Estas memórias são formadas durante a permanência do paciente em UTI e possuem um potencial traumático na medida em que se relacionam à vivência de ameaça de morte, estar gravemente enfermo. A memória traumática é um registro neurológico do trauma, o qual, não é compreendido como uma memória de qualquer tipo de evento estressor, mas, necessariamente, é um registro cognitivo revestido de conteúdo emocional gerado por um evento extremo de ameaça à vida. Alguns estudos sobre o transtorno de estresse pós-traumático (TEPT) em pacientes após UTI classificaram as memórias do período de internação em memórias reais, memórias de sentimentos e memórias ilusórias, sendo que a memórias ilusórias demonstraram forte correlaçáo à formação da memória traumática e TEPT. ${ }^{(1,3,7-9)}$ As memórias ilusórias são memórias de formaçóes delirantes e oníricas, gravadas no momento de recuperação da consciência após sedação e tem sido associadas à característica da doença, tratamento medicamentoso e estado de hipnose produzido por drogas sedativas. ${ }^{(1,3,7)}$ 
O conceito de trauma teve suas bases fundamentadas nos estudos sobre as neuroses de Sigmund Freud, o qual, inicialmente, utilizou o termo para referir as reaçôes psicológicas diante acidentes ferroviários e o impacto da morte em indivíduos veteranos de guerra, sendo aprofundado nos estudos da psique humana. Assim, o termo trauma, até então utilizado para referir acidentes corporais, foi aplicado a fenômenos psicológicos, trazendo ao conceito de traumatismo psicológico a noção de casualidade e tratamento. Posteriormente, os conceitos de trauma e neurose traumática propagaram-se durante a guerra do Vietnã e encontraram solo fértil no movimento contra a violência e os efeitos psicológicos nocivos em populaçóes envolvidas em conflitos armados. Esse movimento culminou na proposta de diagnóstico TEPT na Classificação Diagnóstica Americana em sua terceira versão (Diagnostic and Statistical Manual of Mental Disorders-DSM-III). ${ }^{(10)}$

Atualmente, o sistema diagnóstico mais utilizado na avaliação de TEPT é o publicado na $4^{\mathrm{a}}$ edição da Classificação Diagnóstica Americana (DSM-IV) da American Psychiatric Association e que possui critérios semelhantes aos da Classificação Internacional das Doenças (CID) da Organização Mundial de Saúde (OMS) em sua $10^{a}$ edição. Assim, o TEPT é definido como um conjunto de reaçóes associados à memória do evento traumático, o qual é decorrente de uma vivência de ameaça de morte, por exemplo, acidentes automobilísticos, desastre natural e ser portador de uma doença grave com risco de morte. Outras situaçóes potencialmente traumáticas são consideradas, como a notícia de morte inesperada, ameaça de morte ou lesão experimentada por membro da família ou pessoa próxima, portanto não sofrida diretamente pelo indivíduo. $\mathrm{O}$ indivíduo pode apresentar vivência persistente e intensa das memórias do evento traumático que se manifestam de maneira intrusiva (sem controle do paciente), gerando sofrimento psicológico, manifestaçóes fisiológicas semelhantes às ocorridas durante o evento, comportamento de esquiva e excitabilidade persistentes, podendo apresentar prejuízo ocupacional. Os sintomas habitualmente aparecem depois de um mês e até três meses do evento traumático, configurando um quadro agudo se a sintomatologia estiver presente durante dois dias, um quadro crônico se a sintomatologia persistir por mais de três meses, ou um quadro tardio se a sintomatologia apresentar-se após seis meses. Os sintomas de TEPT podem configurar quadros de reação aguda, crônica e tardia. ${ }^{(11)}$

A prevalência e incidência de TEPT, as variáveis interferentes na concepção do quadro de estresse pós-traumático e as razóes para o desenvolvimento da experiência traumática em pacientes com internação em UTI são temas devidamente documentados. O impacto do TEPT na reabilitação física, saúde mental e social tem sido documentado através da avaliação da qualidade de vida em populações específicas de pacientes que estiveram internados em UTI. Assim, o presente estudo propóe realizar uma revisão sistemática de publicaçóes relevantes dos últimos 12 anos sobre TEPT em pacientes internados em UTI, enfocando os aspectos psicológicos e as intervenções terapêuticas.

\section{MÉTODOS}

A revisão foi realizada a partir de busca em base de dados eletrônica (Medline) nos últimos 12 anos (1996-2008). A estratégia de pesquisa incluiu as palavras-chave: "psychology or psychoterapy" e "posttraumatic stress disorders and intensive care", retirados da seleção os trabalhos relacionados a cuidados pediátrico e neonatal. Os artigos foram incluídos após a concordância entre dois avaliadores que analisaram títulos, resumos e os artigos na integra.

\section{RESULTADOS}

Após a revisão dos 117 artigos, 78 foram excluídos por (a) não serem realizados com pacientes internados em UTI, (b) serem artigos com pacientes pediátricos, (c) cartas de comunicação e (d) método qualitativo com descrição de 1 caso clínico. Os 38 artigos incluídos utilizaram os seguintes desenhos: 9 transversais, 15 longitudinais, 5 caso-controle, 8 revisóes de literatura e 1 estudo qualitativo.

Todos os estudos avaliados utilizaram o DSM - IV Diagnostic and Statistical Manual of Mental Disorders (Fourth Edition Revised) para a definição e caracterização do transtorno.

\section{Prevalência e incidência de transtorno de estresse pós-traumático}

Estudos demonstraram prevalência do TEPT na populaçáo de pacientes internados em UTI geral entre 17\% a $30 \%,{ }^{(9,12,13)}$ variação que pode ser possivelmente atribuída aos diferentes instrumentos de avaliação. Em amostra específicas de pacientes a variação foi menor, $24 \%$ em pacientes com peritonite secundária, ${ }^{(14)} 14 \%$ a $24 \%$ em pacientes com traumas físicos devido a acidente de veículos motorizados e quedas ${ }^{(15-18)}$ e $23 \%$ em pacientes que realizaram transplante de fígado (Quadro 1). ${ }^{(19)}$

As taxas de incidência de TEPT apresentaram pouca variação nas amostras específicas e provenientes de UTI geral: $14 \%$ a $18 \%$ em pacientes cardíacos graves; ${ }^{(20,21)} 24 \%$ em pacientes traumatizados; ${ }^{(22)} 15 \%$ a $24 \%$ após 3 meses e $20 \%$ após 9 meses e 12 meses da alta de UTI geral. ${ }^{(23-25)}$ Por outro lado, alguns estudos com amostras de pacientes 
Quadro 1 - Estudos de prevalência de transtorno de estresse pós-traumático em pacientes após unidade de terapia intensiva

\begin{tabular}{|c|c|c|c|c|c|c|c|c|}
\hline Estudo & Populaçáo & Objetivo & Desenho & Amostra & Instrumentos & Avaliação & $\begin{array}{l}\text { Taxa de } \\
\text { TEPT }\end{array}$ & $\begin{array}{c}\text { Fatores de risco } \\
\text { para TEPT }\end{array}$ \\
\hline $\begin{array}{l}\text { Scragg et al. } \\
(2001)^{(9)}\end{array}$ & UTI geral & Prevalência & Transversal & $\begin{array}{l}142 \text { total } \\
86 \text { final }\end{array}$ & $\begin{array}{c}\text { IES } \\
\text { HADS } \\
\text { TSC-33 }\end{array}$ & $\begin{array}{l}24 \text { meses } \\
\text { (de 1995 } \\
\text { a 1997) }\end{array}$ & $\begin{array}{c}30 \% \text { na } \\
\text { alta }\end{array}$ & $\begin{array}{l}\text { Gênero femini- } \\
\text { no, jovens, ansie- } \\
\text { dade e depressão }\end{array}$ \\
\hline $\begin{array}{l}\text { Nickel et al. } \\
(2004)^{(12)}\end{array}$ & UTI geral & Prevalência & Transversal & $\begin{array}{l}217 \text { total } \\
125 \text { final }\end{array}$ & $\begin{array}{c}\text { PTSS-10 } \\
\text { SCID - DSM-IV }\end{array}$ & $\begin{array}{l}12 \text { meses } \\
(1999)\end{array}$ & $17 \%$ & $\begin{array}{l}\text { Depressáo, alcoo- } \\
\text { lismo, personali- } \\
\text { dade borderline }\end{array}$ \\
\hline $\begin{array}{l}\text { Cuthbertson } \\
\text { et al. } \\
(2004)^{(13)}\end{array}$ & UTI geral & Prevalência & Transversal & $\begin{array}{l}111 \text { total } \\
78 \text { final }\end{array}$ & $\begin{array}{c}\text { DTS } \\
\text { DSM-IV }\end{array}$ & $\begin{array}{l}3 \text { meses } \\
\text { após alta } \\
\text { UTI }\end{array}$ & $22 \%$ & Pacientes jovens \\
\hline $\begin{array}{l}\text { Schnyder et } \\
\text { al. }(2000)^{(15)}\end{array}$ & $\begin{array}{c}\text { Pacientes } \\
\text { vítimas de } \\
\text { acidentes com } \\
\text { trauma severo }\end{array}$ & Prevalência & Longitudinal & 106 total & $\begin{array}{c}\text { IES } \\
\text { CA PTSD Scale } \\
\text { SCQ } \\
\text { FQCI }\end{array}$ & $\begin{array}{l}\text { Média de } \\
14 \text { dias } \\
\text { após } \\
\text { acidente }\end{array}$ & $20 \%$ & $\begin{array}{c}\text { Gênero feminino, } \\
\text { percepçáo subje- } \\
\text { tiva da severidade } \\
\text { da do acidente e } \\
\text { estratégias depres- } \\
\text { sivas em enfren- } \\
\text { tar situação de } \\
\text { estresse }\end{array}$ \\
\hline $\begin{array}{l}\text { Schnyder } \\
\text { et al. } \\
(2001)^{(16,21)}\end{array}$ & $\begin{array}{c}\text { Pacientes } \\
\text { vítimas de } \\
\text { acidentes com } \\
\text { trauma severo }\end{array}$ & Prevalência & Longitudinal & 106 total & $\begin{array}{c}\text { IES } \\
\text { HADS } \\
\text { SNI } \\
\text { IEL } \\
\text { SCQ } \\
\text { FQCI }\end{array}$ & 12 meses & $14 \%$ & $\begin{array}{l}\text { Ansiedade, de- } \\
\text { pressão, percep- } \\
\text { ção de morte }\end{array}$ \\
\hline $\begin{array}{l}\text { Richter et al. } \\
(2006)^{(17)}\end{array}$ & $\begin{array}{c}\text { Pacientes } \\
\text { vítimas de } \\
\text { acidentes com } \\
\text { trauma severo }\end{array}$ & Prevalência & Transversal & $\begin{array}{l}101 \text { total } \\
37 \text { final }\end{array}$ & DSM-IV & $\begin{array}{c}4 \text { anos } \\
\text { após UTI }\end{array}$ & $24 \%$ & $\begin{array}{l}\text { Paciente com } \\
\text { trauma }\end{array}$ \\
\hline $\begin{array}{l}\text { Rothenhäusler } \\
\text { et al. }(2002)^{(19)}\end{array}$ & $\begin{array}{l}\text { Transplante } \\
\text { de fígado }\end{array}$ & Prevalência & Transversal & $\begin{array}{l}81 \text { total } \\
75 \text { final }\end{array}$ & $\begin{array}{c}\text { PTSS-10 } \\
\text { SCID - DSM-IV } \\
\text { SKT } \\
\text { MADRS } \\
\text { SF-36 }\end{array}$ & $\begin{array}{l}48 \text { meses } \\
\text { (de } 1996 \\
\text { a } 1997)\end{array}$ & $23 \%$ & $\begin{array}{c}\text { Depressão e re- } \\
\text { jeição aguda do } \\
\text { órgão }\end{array}$ \\
\hline $\begin{array}{l}\text { Boer et al. } \\
(2007)^{(14)}\end{array}$ & $\begin{array}{l}\text { Peritonite } \\
\text { secundária - } \\
\text { cirúrgica }\end{array}$ & Prevalência & Transversal & $\begin{array}{l}278 \text { total } \\
118 \text { vivos } \\
104 \text { final }\end{array}$ & PTSS-10 & $\begin{array}{l}96 \text { meses } \\
(\text { de } 1994 \\
\text { à 2000) }\end{array}$ & $24 \%$ & $\begin{array}{c}\text { Mulheres acima } \\
\text { de } 50 \text { anos }\end{array}$ \\
\hline
\end{tabular}

TEPT - transtorno de estresse pós-traumático; UTI - unidade de terapia intensive; IES - Impact Event Stressor; HADS - Hospital Anxiety and Depression Scale; TSC-33 - Trauma Symtom Checklist-33; PTSS - Post-traumatic Stress Syndrome 10-questions Inventory; SCID - Strutural Clinical Interview for the DSM-IV; DTS - Davidson Trauma Scale; DSM-IV, Diagnostic and Statistical Manual of Mental Disorders - IV; CA PTSD Scale - ClinicalAdministered PTSD Scale; SCQ - Sense Coherence Questionnaire; FQCI - Freiburg Questionnaire of Coping with Illness; SNI - Social Network Index; ILE - Inventory for Determining Life-Changing Events; SKT - Syndrom-Kurztest; MADRS - Montgomery-Asberg Depression Rating Scale; SF-36 - Medical Outcomes Study 36-Item Short Form.

de UTI geral revelaram baixa incidência. Samuelson et al. apresentaram uma taxa de 8,5\% para TEPT agudo e Capuzzo et al. encontraram apenas $5 \%$ de TEPT, sendo que neste último estudo foram excluídos os pacientes com tratamento prévio de depressão e ansiedade com uso de antidepressivos e neurolépticos, considerados fatores de risco para TEPT (Quadro 2). ${ }^{(26,27)}$

Scragg et al. referiram que a variação das taxas de pre- valência e incidência podem ser atribuídas aos diferentes instrumentos de avaliação aplicados (Impact Event Scale Revised; SCID - Strutural Clinical Interview for the DSM-IV; Structural Clinical Interview; Posttraumatic Stress Syndrome 10-Questions Inventory), mesmo tendo apresentado confiabilidade e validade aceitáveis para as amostras estudadas. ${ }^{(9)}$ Outros estudos consideraram que a variação das taxas pode ser atribuída também aos diferentes momentos de aplicação 
Quadro 2 - Estudos de incidência de transtorno de estresse pós-traumático em pacientes após unidade de terapia intensiva

\begin{tabular}{|c|c|c|c|c|c|c|c|c|}
\hline Estudo & População & Objetivo & Desenho & Amostra & Instrumentos & Avaliação & $\begin{array}{l}\text { Taxa de } \\
\text { TEPT }\end{array}$ & $\begin{array}{c}\text { Fatores de risco } \\
\text { para TEPT }\end{array}$ \\
\hline $\begin{array}{l}\text { Capuzzo et al. } \\
(2005)^{(26)}\end{array}$ & UTI geral & Incidência & Longitudinal & $\begin{array}{l}84 \text { total } \\
63 \text { final }\end{array}$ & $\begin{array}{c}\text { IES } \\
\text { ICU-MT }\end{array}$ & $\begin{array}{c}1 \text { semana } \\
\text { e } 3 \text { meses } \\
\text { após alta da } \\
\text { UTI }\end{array}$ & $5 \%$ & $\begin{array}{c}\text { Memórias } \\
\text { ilusórias }\end{array}$ \\
\hline $\begin{array}{l}\text { Rattray et al. } \\
(2005)^{(23)}\end{array}$ & UTI geral & Incidência & Longitudinal & $\begin{array}{l}109 \text { total } \\
80 \text { final }\end{array}$ & $\begin{array}{l}\text { ICEQ } \\
\text { HADS } \\
\text { IES }\end{array}$ & $\begin{array}{l}\text { No mo- } \\
\text { mento de } \\
\text { alta do } \\
\text { hospital, } 6 \\
\text { meses e } 12 \\
\text { meses após }\end{array}$ & $\begin{array}{l}\text { Evitação: } \\
20 \% \text { - } \\
12 \text { meses } \\
\text { Intrusão: } \\
18 \% \text { - } \\
12 \text { meses }\end{array}$ & $\begin{array}{l}\text { Ansiedade, de- } \\
\text { pressão e tempo } \\
\text { de internaçáo } \\
\text { associado com } \\
\text { sintomas após } \\
12 \text { meses }\end{array}$ \\
\hline $\begin{array}{l}\text { Sukantarat et } \\
\text { al. }(2007)^{(24)}\end{array}$ & UTI geral & Incidência & Longitudinal & $\begin{array}{l}51 \text { total } \\
45 \text { final }\end{array}$ & $\begin{array}{c}\text { IES } \\
\text { HADS } \\
\text { SF-36 }\end{array}$ & $\begin{array}{c}3 \text { e } 9 \text { meses } \\
\text { após alta }\end{array}$ & $\begin{array}{l}24 \%- \\
3 \text { meses } \\
20 \%- \\
9 \text { meses }\end{array}$ & $\begin{array}{c}\text { Ansiedade, } \\
\text { depressão e } \\
\text { declínio físico }\end{array}$ \\
\hline $\begin{array}{l}\text { Samuelson et } \\
\text { al. }(2007)^{(27)}\end{array}$ & UTI geral & Incidência & Longitudinal & $\begin{array}{l}313 \text { total } \\
226 \text { final }\end{array}$ & $\begin{array}{l}\text { IES-R } \\
\text { HADS } \\
\text { ICU-MT } \\
\text { ICU-SEQ }\end{array}$ & $\begin{array}{c}5 \text { dias e } 2 \\
\text { meses após } \\
\text { alta de UTI }\end{array}$ & $\begin{array}{l}8.5 \% \text { TEPT } \\
\text { agudo }\end{array}$ & $\begin{array}{c}\text { Ansiedade e } \\
\text { depressão }\end{array}$ \\
\hline $\begin{array}{l}\text { Jones et al. } \\
(2007)^{(25)}\end{array}$ & UTI geral & Incidência & Longitudinal & $\begin{array}{l}799 \text { total } \\
238 \text { final }\end{array}$ & $\begin{array}{l}\text { PTSS-10 } \\
\text { ICU-MT }\end{array}$ & $\begin{array}{l}2 \text { semanas, } \\
2 \text { meses e } 3 \\
\text { meses }\end{array}$ & $\begin{array}{c}15 \%-3 \\
\text { meses }\end{array}$ & $\begin{array}{l}\text { Memórias ilu- } \\
\text { sórias, sedação } \\
\text { prolongada e } \\
\text { contenção física } \\
\text { sem sedação }\end{array}$ \\
\hline $\begin{array}{l}\text { Rothenhäusler } \\
\text { et al. } \\
(2005)^{(20)}\end{array}$ & $\begin{array}{l}\text { Pacientes } \\
\text { cardíacos }\end{array}$ & Incidência & Longitudinal & 34 total & $\begin{array}{c}\text { PTSS-10 } \\
\text { SCID - DSM-IV } \\
\text { SKT } \\
\text { MADRS } \\
\text { SF-36 } \\
\text { DRS }\end{array}$ & $\begin{array}{l}\text { Em UTI e } \\
12 \text { meses } \\
\text { após alta }\end{array}$ & $18 \%$ & $\begin{array}{l}\text { Ansiedade e } \\
\text { depressão }\end{array}$ \\
\hline $\begin{array}{l}\text { Girard et al. } \\
(2007)^{(21)}\end{array}$ & $\begin{array}{l}\text { Pacientes } \\
\text { cardíacos }\end{array}$ & Incidência & Longitudinal & $\begin{array}{l}275 \text { total } \\
43 \text { final }\end{array}$ & PTSS-10 & $\begin{array}{l}6 \text { meses } \\
\text { após alta }\end{array}$ & $14 \%$ & $\begin{array}{c}\text { Mulheres, jovens } \\
(\leq 50) \text {, alta dose } \\
\text { de lorazepam }\end{array}$ \\
\hline $\begin{array}{l}\text { Matsuoka et } \\
\text { al. }(2008)^{(22)}\end{array}$ & $\begin{array}{l}\text { Pacientes } \\
\text { vítimas de } \\
\text { acidente } \\
\text { de veículo } \\
\text { motori- } \\
\text { zado }\end{array}$ & Incidência & Longitudinal & $\begin{array}{l}188 \text { total } \\
100 \text { final }\end{array}$ & $\begin{array}{c}\text { IES-R } \\
\text { CA PTSD Scale } \\
\text { HADS } \\
\text { MINI }\end{array}$ & $\begin{array}{l}4 \text { a } 6 \text { sema- } \\
\text { nas após } \\
\text { acidente }\end{array}$ & $24 \%$ & $\begin{array}{l}\text { Percepção de } \\
\text { risco de vida, } \\
\text { taxa cardíaca e } \\
\text { subescala de me- } \\
\text { mória intrusiva } \\
\text { da IES-R }\end{array}$ \\
\hline
\end{tabular}

TEPT - transtorno de estresse pós-traumático; UTI - unidade de terapia intensive; IES - Impact Event Stressor; ICU-MT- Intensive Care Unit - Memory Tool; ICEQ - Intensive Care Experience Questionnaire; HADS - Hospital Anxiety and Depression Scale; SF-36 - Medical Outcomes Study 36-Item Short Form; ICU-SEQ - Intensive Care Unit, Stressful Experience Questionnaire; PTSS - Post-traumatic Stress Syndrome 10-questions Inventory; SCID - Strutural Clinical Interview for the DSM-IV; SKT- Syndrom-Kurztest; MADRS - Montgomery-Asberg Depression Rating Scale; DRS - Delirium Rating Scale; IES-R - Impact Event Stressor-Revised; CA PTSD Scale - Clinical-Administered PTSD Scale; MINI - Mini-International Neuropsychiatric Interview.

dos instrumentos e ao tamanho e especificidade diferentes das amostras avaliadas nos estudos citados. ${ }^{(28,29)}$ A diferença no procedimento da sedação dos pacientes em UTI, prática diferenciada entre países e serviços, também foi apontada como sendo uma variável de risco importante para o TEPT, podendo influenciar as taxas. ${ }^{(26,30)}$

\section{Fatores de risco}

Os fatores de risco associados ao TEPT referidos foram (a) história prévia de ansiedade, depressão ou pânico, ${ }^{(12,31)}$ (b) presença de memórias ilusórias ${ }^{(3,12,31,32)}$ (c) presença de crenças e de comportamentos depressivos que impossibilitam o enfrentamento da situaçáo de ameaça, ${ }^{(15)}(\mathrm{d})$ experiências es- 
tressantes como pesadelos e sentimentos de medo ${ }^{(27,33)} \mathrm{e}(\mathrm{e})$ tempo de ventilação mecânica. ${ }^{(13,31)}$

A presença de sintomas de ansiedade e depressão mostrou forte correlaçấo com o desenvolvimento de comportamentos de evitaçáo e de memórias intrusivas (sintomatologia de TEPT) ${ }^{(23,24,31)}$ A presença de memórias ilusórias decorrente da internação em UTI revelou correlação significativa com o aumento do TEPT agudo e crônico, transtorno de ansiedade, comportamentos fóbicos e transtornos de pânico, independente do instrumento de avaliação e do momento em que a avaliação foi realizada. . $^{(3,5,12,13,27,32)}$

Drogas sedativas e as respectivas dosagens foram estudas como fatores de risco para o desenvolvimento de delirium e conseqüente formação de memórias ilusórias em pacientes internados em UTI. Pacientes mais propensos a apresentar delirium foram (a) pacientes que receberam doses diárias altas de opióides (dose mediana de $88 \mathrm{mg} /$ dia vs $43 \mathrm{mg} /$ dia; $\mathrm{p}=0,039$ ) e (b) pacientes que apresentaram sintomas de abstinência da sedação e analgesia (78\% da amostra; $\mathrm{p}<0.0001) .{ }^{(25)}$ Os pacientes que receberam doses altas de propofol como sedativo não apresentaram delirium. ${ }^{(25)} \mathrm{O}$ total de dose recebido por pacientes em UTI do sedativo lorazepam também foi associado ao TEPT; para cada aumento de $10 \mathrm{mg}$ de lorazepam encontrou-se um aumento de 0,39 no escore total do instrumento de avaliação de sintomas de TEPT (Posttraumatic Stress Syndrome 10-Questions Inventory) (IC95\% 0,17 - 0,61; $\mathrm{p}=0,04) .{ }^{(21)}$

Ainda referente à sedação, os procedimentos de sedação contínua ou diária (retirada diária de sedação) foram referidos como fatores de risco e de proteçấo. Os pacientes que receberam sedação contínua e profunda apresentaram memórias ilusórias e poucas memórias reais sobre seu tratamento no período da sedação, portanto estiveram suscetíveis ao desenvolvimento de sintomatologia de TEPT. ${ }^{(3,9)} \mathrm{O}$ que ocorre de maneira inversa quando os pacientes foram submetidos ao procedimento diário de sedação. ${ }^{(26,30)}$ As variáveis (a) tempo de internação em UTI, (b) diagnóstico inicial, (c) gravidade da doença e (d) duração de delirium náo mostraram associaçâo como escore geral de ansiedade, depressão, estresse ou TEPT. ${ }^{(3,5,12,13,21,23,32,34)}$

A associação entre idade e gênero e o TEPT mostrou-se controversa. Referente a gênero alguns estudos não demonstraram relação com a sintomatologia de TEPT. ${ }^{(3,5,12,13,32,32)}$ Por outro lado, outros estudos apresentaram associação de gênero com a sintomatologia de TEPT sendo a proporção da manifestação do TEPT nas mulheres maior do que nos homens. ${ }^{(21,26,27,34)} \mathrm{A}$ idade dos pacientes não mostrou associação com o TEPT em alguns estudos. ${ }^{(3,5,12,32)}$ Em outros estudos: o TEPT esteve correlacionado inversamente com a idade; ${ }^{(13,31)}$ pacientes após 50 anos apresentaram menos sintomas de TEPT; ${ }^{(21)}$ pacientes jovens apresentaram maiores escores de ansiedade e depressão após 6 e 12 meses da alta hospitalar, bem como maiores níveis de sintomas comportamentais de evitação e memórias intrusivas estiveram presentes na alta hospitalar, após 6 meses e após 12 meses; ${ }^{(23)}$ e nível grave de TEPT foi encontrado em jovens. ${ }^{(27)}$

\section{O impacto do transtorno de estresse pós-traumático na saúde global}

O impacto do TEPT na reabilitação física, saúde mental e social foi estudado por meio da qualidade de vida e estudo qualitativo em pacientes que estiveram internados em UTI.

Estudo realizado com amostra de pacientes com síndrome respiratória aguda avaliou a qualidade de vida através do instrumento Medical Outcomes Study 36 Iten Short Form (SF-36) e encontrou diferença significativa entre os grupos com e sem TEPT nas dimensóes saúde mental $(\mathrm{F}=54.11 ; \mathrm{df}=2 ; \mathrm{p}=0,0001)$, aspectos físicos $(\mathrm{F}=36.81$; $\mathrm{df}=2 ; \mathrm{p}=0,0001)$, capacidade funcional $(\mathrm{F}=17.92 ; \mathrm{df}=2$; $\mathrm{p}=0,0001)$, dor $(\mathrm{F}=35.81 ; \mathrm{df}=2 ; \mathrm{p}=0,0001)$ e estado geral de saúde $(\mathrm{F}=37.52 ; \mathrm{df}=2 ; \mathrm{p}=0,0001) .{ }^{(34)}$ Resultados semelhantes foram encontrados por Schelling et al. (1998) e Kapfhammer et al. nas seguintes dimensões respectivamente: saúde mental $(-20 \% ; \mathrm{p}=0,001)$, $(\mathrm{H}=11.92 ; \mathrm{p}=0,003)$; vitalidade $(-17 \% ; \mathrm{p}=0,002)$; aspectos sociais $(-13 \% ; \mathrm{p}=0,046),(\mathrm{H}=10.75 ; \mathrm{p}=0,005)$ e maior freqüência de dor $(+27 \% ; \mathrm{p}=0,002)$ e estado geral de saúde $(\mathrm{H}=12.11 ; \mathrm{p}=0,002) .^{(5,35)}$ Estudo com amostra de pacientes cardíacos após cirurgia apresentou queda da saúde mental $(\mathrm{R}=0,52 ; \mathrm{p}<0,01) .{ }^{(2)}$ Estes dados evidenciam o impacto do TEPT na reabilitação de pacientes crônicos (Quadro 3).

Outros estudos de ensaio clínico e revisão bibliográfica demonstraram que pacientes críticos com insuficiência de corticoesteróides, que sofreram choque séptico e cirurgia cardíaca, foram beneficiados do tratamento com hidrocortisona, sendo que o grupo tratamento quando comparado ao grupo controle apresentou significante melhora da sintomatologia de TEPT, da qualidade de vida e de reabilitação. $^{(4,5,36)}$

Estudo qualitativo fenomenológico das reaçóes de estresse pós-traumático em pacientes após internação em UTI revelou que a internaçáo foi vivida como uma situaçáo de vida fora de controle, onde sentimentos de impotência e o medo do desconhecido geraram experiências de profundo impacto jamais vivido e repercussóes ao longo da reabilitação. As variaçóes fenomenológicas foram relacionadas aos efeitos das memórias traumáticas, como (a) ser assombrado pelo trauma, (b) necessidade de escapar, (c) aflição e tensão em relação à situaçáo de vida, e (d) poder ser transformado e afetado pelo conteúdo da memória. ${ }^{(37)}$ 
Quadro 3 - Estudos do impacto de transtorno de estresse pós-traumático na qualidade de vida de pacientes após unidade de terapia intensiva

\begin{tabular}{|c|c|c|c|c|c|c|c|}
\hline Estudo & Populaçáo & Desenho & Amostra & Instrumentos & Tempos de avaliação & $\begin{array}{l}\text { Taxa de } \\
\text { TEPT }\end{array}$ & $\begin{array}{l}\text { Qualidade de vida com } \\
\text { TEPT }\end{array}$ \\
\hline $\begin{array}{l}\text { Schelling et } \\
\text { al. }(1998)^{(5)}\end{array}$ & $\begin{array}{l}\text { Síndrome } \\
\text { respiratória } \\
\text { aguda }\end{array}$ & Transversal & $\begin{array}{l}192 \text { total } \\
80 \text { final }\end{array}$ & $\begin{array}{l}\text { DSM - III } \\
\text { SF-36 } \\
\text { PTSS-10 }\end{array}$ & $\begin{array}{l}\text { Pacientes atendidos } \\
\text { de } 1985 \text { a } 1995\end{array}$ & $41 \%$ na alta & $\begin{array}{l}\text { Declínio da saúde mental, } \\
\text { função física, função social } \\
\text { e maior freqüência de dor }\end{array}$ \\
\hline $\begin{array}{l}\text { Kapfhammer } \\
\text { et al. } \\
(2004)^{(35)}\end{array}$ & $\begin{array}{l}\text { Síndrome } \\
\text { respiratória } \\
\text { aguda }\end{array}$ & Transversal & 80 total & $\begin{array}{l}\text { PTSS-10 } \\
\text { MADRS } \\
\text { STAI X1 } \\
\text { SSD } \\
\text { SKT } \\
\text { SAQSS } \\
\text { SF-36 }\end{array}$ & $\begin{array}{l}\text { Média de } 8 \text { anos } \\
\text { após UTI }\end{array}$ & $\begin{array}{l}\text { 43\% na alta } \\
\text { da UTI; } \\
\text { 14\% } 8 \text { anos } \\
\text { após UTI }\end{array}$ & $\begin{array}{l}\text { Declínio da saúde mental, } \\
\text { funçáo social, percepçáo } \\
\text { geral da saúde }\end{array}$ \\
\hline $\begin{array}{l}\text { Deja et al. } \\
(2006)^{(34)}\end{array}$ & $\begin{array}{l}\text { Síndrome } \\
\text { respiratória } \\
\text { aguda }\end{array}$ & Transversal & $\begin{array}{l}263 \text { total } \\
65 \text { final }\end{array}$ & $\begin{array}{l}\text { SF-36 } \\
\text { PTSS-10 } \\
\text { F-Sozu } \\
\text { SC-90-R }\end{array}$ & $\begin{array}{l}\text { Pacientes atendidos } \\
\text { de } 1991 \text { a } 2000- \\
\text { início da coleta } 2002\end{array}$ & $29 \%$ na alta & Declínio da saúde mental \\
\hline $\begin{array}{l}\text { Schelling et } \\
\text { al. }(2003)^{(2)}\end{array}$ & $\begin{array}{l}\text { Pacientes } \\
\text { cardíacos }\end{array}$ & Longitudinal & $\begin{array}{l}387 \text { total } \\
148 \text { final }\end{array}$ & $\begin{array}{l}\text { DSM - IV } \\
\text { SF-36 }\end{array}$ & $\begin{array}{l}\text { Antes da cirurgia, } \\
1 \text { dia após cirurgia, } \\
1 \text { semana e } 6 \text { meses } \\
\text { após alta da UTI }\end{array}$ & $\begin{array}{l}18,2 \% \text { após } \\
6 \text { meses }\end{array}$ & $\begin{array}{l}\text { Declínio da saúde mental } \\
\text { e sem percepção de ga- } \\
\text { nhos na condição física } \\
\text { após cirurgia }\end{array}$ \\
\hline
\end{tabular}

TEPT - transtorno de estresse pós-traumático; UTI - unidade de terapia intensiva; DSM - III - Diagnostic and Statistical Manual of Mental Disorders - III; DSM - IV - Diagnostic and Statistical Manual of Mental Disorders - IV; SF-36 - Medical Outcomes Study 36-Item Short Form; PTSS - Posttraumatic Stress Syndrome 10-questions Inventory; MADRS - Montgomery-Asberg Depression Rating Scale; STAI X1 - State-Trait Anxiety Inventory X1; SSD - Screening Somatoform Disorders; SKT - Syndrom-Kurztest; SAQSS - Self-Assessment Questionnaire on Social Support, F-Sozu - Questionnaire for Social Support; SC-90-R - Symptom Checklist-90-R.

\section{Tratamentos}

Os estudos de tratamento do TEPT na UTI descreveram que a administração de hidrocortisona pode resultar em menor freqüência de aparecimento de sintomas de TEPT ou de sintomas de estresse crônico; porém, não produziu efeito sobre a formação, nem sobre o número de memórias traumáticas. A administração de hidrocortisona introduzida no período pré-operatório de cirurgia cardíaca seguiu os seguintes parâmetros: $100 \mathrm{mg}$ por 10 minutos de hidrocortisona, antes da anestesia; infusão contínua de $10 \mathrm{mg} /$ hora por 24 horas no primeiro dia do pós-operatório; $5 \mathrm{mg} /$ hora no segundo dia do pós-operatório; 3 vezes ao dia de $20 \mathrm{mg}$ no terceiro dia do pós-operatório; 3 vezes ao dia de $10 \mathrm{mg}$ no quarto dia do pós-operatório. Os autores demonstraram que a hidrocortisona inibiu a formação de memórias intrusivas, mas os mecanismos deste efeito não foram evidenciados. ${ }^{(1,4,6-8,36,38)}$

$\mathrm{O}$ uso de bloqueadores beta-adrenérgico diminuiu a freqüência das memórias traumáticas em pacientes após cirurgia cardíaca, porém este resultado não foi estatisticamente significante. ${ }^{(2)}$ Pacientes do grupo tratamento que receberam hormônio noroepinefrina durante a cirurgia cardíaca demonstraram uma significativa diminuição na sintomatologia de TEPT e melhora na qualidade de vida, quando comparados ao grupo controle. ${ }^{(6)} \mathrm{O}$ uso de epinefrina, ao contrário, apresentou aumento das memórias ilusórias traumáticas e índices elevados de estresse e TEPT. ${ }^{(2)}$

Kress et al. investigaram efeitos psicológicos do procedimento de interrupçáo diária da sedaçáo e constataram que os pacientes que não foram submetidos a este procedimento tiveram mais memórias ilusórias e significativa diminuição na sintomatologia de TEPT (11.2 vs. 27.3, p=0,02). Os autores sugerem que a memória real da experiência da UTI proporcionada pela interrupção diária da sedação pode ser fator protetor. ${ }^{(30)} \mathrm{A}$ sedação diária demonstrou benefícios aos pacientes, como alterar os processos de memorizaçáo, diminuir a quantidade de medicaçáo e do tempo de ventilação mecânica, sendo que estes fatores podem estar no desenvolvimento de sintomas de TEPT. ${ }^{(39)}$

Uma intervenção cognitiva comportamental foi realizada através da aplicaçáo de um manual educativo enviado aos pacientes após alta hospitalar. O manual continha informaçóes explicativas e sugestivas de enfrentamento diante o aparecimento de sintomas como ansiedade, depressão, transtorno de estresse pós-traumático e sintomas de recuperação física. Houve uma reduçáo do aparecimento de sintomas depressivos $(\mathrm{F}=10.47 ; \mathrm{df}=1 ; \mathrm{p}=0,004)$, porém sem diferença na manifestaçâo de sintomas de ansiedade e de TEPT. ${ }^{(39)}$ 


\section{CONSIDERAÇÓES FINAIS}

Os estudos evidenciam que pacientes que estiveram internados em UTI passam por uma experiência de grande potencial traumático e que uma parte importante destes desenvolve quadros emocionais graves, incluindo o transtorno de estresse pós-traumático. Estas experiências são acompanhadas de um sofrimento psicológico e que repercute de forma prejudicial na reabilitação do paciente. Contudo, as taxas de prevalência e incidência de TEPT são controversas, isso porque existem algumas deficiências na padronizaçáo metodológica dos estudos, entre elas, os diferentes métodos diagnósticos e um grande número de amostras de pacientes com doenças específicas avaliados em UTIs de especialidades. Todos estes fatores dificultam a análise comparativa entre os resultados. Portanto, atualmente existe pouco conhecimento sobre os fatores associados ao desenvolvimento do TEPT e dos tratamentos recebidos pelos pacientes de UTI. ${ }^{(28)}$

Estudos recomendam intervençóes medicamentosas como o uso da hidrocortisona como profilaxia no tratamento do estresse crônico e do TEPT. A hidrocortisona inibe a manifestação intrusiva das memórias, porém não interfere na formação das memórias traumáticas.

A necessidade de cuidados aos pacientes críticos e estratégias de suporte é enfatizada pela literatura. Estes cuidados visam prevenir e aliviar o sofrimento gerado pela experiência do TEPT, portanto, o treinamento de equipes de saúde na identificação do TEPT e no desenvolvimento de intervenção global é recomendável. ${ }^{(32,40)}$

Não há evidências de efetividade de tratamentos psicoterápicos na prevenção de TEPT em pacientes internados em UTI. Apenas um estudo de intervenção psicológica utilizando o referencial cognitivo-comportamental foi realizado e este não demonstrou efetividade na redução de sintomas de TEPT. ${ }^{(32)}$ Novos estudos de avaliaçáo da eficácia de intervençôes psicoterápicas na prevenção e tratamento de TEPT são recomendados.

Agradecimentos: Projeto financiado pela Fundação de Amparo à Pesquisa do Estado de São Paulo (FAPESP), processo no 05/55513-6.

A autora agradece a concessão de bolsa de doutorado da Coordenação de Aperfeiçoamento de Pessoal de Nível Superior e a equipe do Núcleo de Estatística e Metodologia Aplicada (NEMAP) do centro de Estudos do Departamento de Psiquiatria da Universidade Federal de São Paulo.

\section{ABSTRACT}

Post-traumatic stress disorder has been detected in patients after treatment in intensive care unit. The main goal of this study is to review the psychological aspects and therapeutic interventions on those patients after their treatment on intensive care unit. Thirty eight articles have been included. The prevalence of post-traumatic stress disorder has varied from $17 \%$ up to $30 \%$ and the incidence from $14 \%$ to $24 \%$. The risk factors were: previous anxiety historic, depression or panic, having delusional traumatic memories (derived from psychic formations as dreams and delirium), belief effects, depressive behavior, stressing experiences and mechanical ventilation. High doses of opiates, symptoms caused by sedation or analgesia reduction and the use of lorazepam were related with the increase of delirium and delusional memory. The disorder sintomatology can be reduced with hydrocortisone administration, with daily sedation interruption. No other effectiveness psychological intervention study was found.

Keywords: Posttraumatic stress disorder/psychology; Intensive care units; Adult

\section{REFERÊNCIAS}

1. Schelling G, Roozendaal B, De Quervain DJ. Can posttraumatic stress disorder be prevented with glucocorticoids? Ann N Y Acad Sci. 2004;1032:158-66.

2. Schelling G, Richter M, Roozendaal B, Rothenhäusler HB, Krauseneck T, Stoll C, et al. Exposure to high stress in the intensive care unit may have negative effects on health-related quality-of-life outcomes after cardiac surgery. Crit Care Med. 2003;31(7):1971-80.

3. Jones C, Griffiths RD, Humphris G, Skirrow PM. Memory, delusions, and the development of acute posttraumatic stress disorder-related symptoms after intensive care. Crit Care Med. 2001;29(3):573-80.

4. Schelling G, Stoll C, Kapfhammer HP, Rothenhäusler HB, Krauseneck T, Durst K, et al. The effect of stress doses of hydrocortisone during septic shock on posttraumatic stress disorder and health-related quality of life in survivors. Crit Care Med. 1999;27(12):2678-83.

5. Schelling G, Stoll C, Haller M, Briegel J, Manert W, Hummel T, et al. Health-related quality of life and posttraumatic stress disorder in survivors of the acute respiratory distress syndrome. Crit Care Med. 1998;26(4):651-9.

6. Weis F, Kilger E, Roozendaal B, de Quervain DJ, Lamm P, Schmidt M, et al. Stress doses of hydrocortisone reduce chronic stress symptoms and improve health-related quality of life in high-risk patients after cardiac surgery: a randomized study. J Thorac Cardiovasc Surg. 2006;131(2):277-82.

7. Griffiths RD, Jones C. Delirium, cognitive dysfunction and posttraumatic stress disorder. Curr Opin Anaesthesiol. 2007;20(2):124-9. Review.

8. Schelling G, Kilger E, Roozendaal B, de Quervain DJ, Briegel J, Dagge A, et al. Stress doses of hydrocortisone, traumatic memories, and symptoms of posttraumatic stress disorder in pa- 
tients after cardiac surgery: a randomized study. Biol Psychiatry. 2004;55(6):627-33.

9. Scragg P, Jones A, Fauvel N. Psychological problems following ICU treatment. Anaesthesia. 200;56(1):9-14.

10. Schestatsky S, Shansis F, Ceitlin LH, Abreu PBS, Hauck S. Historical evolution of the concept of posttraumatic stress disorder. Rev Bras Psiquiatr. 2003;25 Suppl 1:8-11.

11. Kapczinski F, Margis R. Posttraumatic stress disorder: diagnostic criteria. Rev Bras Psiquiatr. 2003;25 Suppl 1:3-7.

12. Nickel M, Leiberich P, Nickel C, Tritt K, Mitterlehner F, Rother $\mathrm{W}$, Loew T. The occurrence of posttraumatic stress disorder in patients following intensive care treatment: a cross-sectional study in a random sample. J Intensive Care Med. 2004;19(5):285-90.

13. Cuthbertson BH, Hull A, Strachan M, Scott J. Post-traumatic stress disorder after critical illness requiring general intensive care. Intensive Care Med. 2004;30(3):450-5.

14. Boer KR, Mahler CW, Unlu C, Lamme B, Vroom MB, Sprangers $\mathrm{MA}$, et al. Long-term prevalence of post-traumatic stress disorder symptoms in patients after secondary peritonitis. Crit Care. 2007;11(1):R30.

15. Schnyder U, Mörgeli H, Nigg C, Klaghofer R, Renner N, Trentz $\mathrm{O}$, et al. Early psychological reactions to life-threatening injuries. Crit Care Med. 2000;28(1):86-92.

16. Schnyder U, Moergeli H, Klaghofer R, Buddeberg C. Incidence and prediction of posttraumatic stress disorder symptoms in severely injured accident victims. Am J Psychiatry. 2001;158(4):594-9.

17. Richter JC, Waydhas C, Pajonk FG. Incidence of posttraumatic stress disorder after prolonged surgical intensive care unit treatment. Psychosomatics. 2006;47(3):223-30.

18. Schnyder U, Moergeli H, Trentz O, Klaghofer R, Buddeberg C. Prediction of psychiatric morbidity in severely injured accident victims at one-year follow-up. Am J Respir Crit Care Med. 2001;164(4):653-6.

19. Rothenhäusler HB, Ehrentraut S, Kapfhammer HP, Lang C, Zachoval R, Bilzer M, et al. Psychiatric and psychosocial outcome of orthotopic liver transplantation. Psychother Psychosom. 2002;71(5):285-97.

20. Rothenhäusler HB, Grieser B, Nollert G, Reichart B, Schelling G, Kapfhammer HP. Psychiatric and psychosocial outcome of cardiac surgery with cardiopulmonary bypass: a prospective 12-month follow-up study. Gen Hosp Psychiatry. 2005;27(1):18-28.

21. Girard TD, Shintani AK, Jackson JC, Gordon SM, Pun BT, Henderson MS, et al. Risk factors for post-traumatic stress disorder symptoms following critical illness requiring mechanical ventilation: a prospective cohort study. Crit Care. 2007;11(1):R28.

22. Matsuoka Y, Nishi D, Nakajima S, Kim Y, Homma M, Otomo $\mathrm{Y}$. Incidence and prediction of psychiatric morbidity after a motor vehicle accident in Japan: the Tachikawa Cohort of Motor Vehicle Accident Study. Crit Care Med. 2008;36(1):74-80.

23. Rattray JE, Johnston M, Wildsmith JA. Predictors of emotional outcomes of intensive care. Anaesthesia. 2005;60(11):1085-92.

24. Sukantarat K, Greer S, Brett S, Williamson R. Physical and psychological sequelae of critical illness. Br J Health Psychol. 2007;12(Pt 1):65-74.

25. Jones C, Bäckman C, Capuzzo M, Flaatten H, Rylander C, Griffiths RD. Precipitants of post-traumatic stress disorder following intensive care: a hypothesis generating study of diversity in care. Intensive Care Med. 2007;33(6):978-85.

26. Capuzzo M, Valpondi V, Cingolani E, Gianstefani G, De Luca S, Grassi L, Alvisi R. Post-traumatic stress disorder-related symptoms after intensive care. Minerva Anestesiol. 2005;71(4):167-79.

27. Samuelson KA, Lundberg D, Fridlund B. Stressful memories and psychological distress in adult mechanically ventilated intensive care patients - a 2-month follow-up study. Acta Anaesthesiol Scand. 2007;51(6):671-8

28. Griffiths J, Fortune G, Barber V, Young JD. The prevalence of post traumatic stress disorder in survivors of ICU treatment: a systematic review. Intensive Care Med. 2007;33(9):1506-18. Review.

29. Jackson JC, Hart RP, Gordon SM, Hopkins RO, Girard TD, Ely EW. Post-traumatic stress disorder and post-traumatic stress symptoms following critical illness in medical intensive care unit patients: assessing the magnitude of the problem. Crit Care. 2007;11(1):R27. Review.

30. Kress JP, Gehlbach B, Lacy M, Pliskin N, Pohlman AS, Hall JB. The long-term psychological effects of daily sedative interruption on critically ill patients. Am J Respir Crit Care Med. 2003;168(12):1457-61.

31. Jackson JC, Obremskey W, Bauer R, Greevy R, Cotton BA, Anderson $\mathrm{V}$, et al. Long-term cognitive, emotional, and functional outcomes in trauma intensive care unit survivors without intracranial hemorrhage. J Trauma. 2007;62(1):80-8.

32. Jones C, Skirrow P, Griffiths RD, Humphris GH, Ingleby S, Eddleston J, et al. Rehabilitation after critical illness: a randomized, controlled trial. Crit Care Med. 2003;31(10):2456-61.

33. Mohta M, Sethi AK, Tyagi A, Mohta A. Psychological care in trauma patients. Injury. 2003;34(1):17-25. Review.

34. Deja M, Denke C, Weber-Carstens S, Schröder J, Pille CE, Hokema F, et al. Social support during intensive care unit stay might improve mental impairment and consequently health-related quality of life in survivors of severe acute respiratory distress syndrome. Crit Care. 2006;10(5):R147.

35. Kapfhammer HP, Rothenhäusler HB, Krauseneck T, Stoll C, Schelling G. Posttraumatic stress disorder and health-related quality of life in long-term survivors of acute respiratory distress syndrome. Am J Psychiatry. 2004;161(1):45-52.

36. Schelling G, Roozendaal B, Krauseneck T, Schmoelz M, DE Quervain D, Briegel J. Efficacy of hydrocortisone in preventing posttraumatic stress disorder following critical illness and major surgery. Ann N Y Acad Sci. 2006;1071:46-53.

37. Corrigan I, Samuelson KA, Fridlund B, Thomé B. The meaning of posttraumatic stress-reactions following critical illness or injury and intensive care treatment. Intensive Crit Care Nurs. 2007;23(4):206-15.

38. Schelling G, Briegel J, Roozendaal B, Stoll C, Rothenhäusler HB, Kapfhammer HP. The effect of stress doses of hydrocortisone during septic shock on posttraumatic stress disorder in survivors. Biol Psychiatry. 2001;50(12):978-85.

39. Meyer NJ, Hall JB. Brain dysfunction in critically ill patients the intensive care unit and beyond. Crit Care. 2006;10(4):223.

40. Lennmarken C, Sydsjo G. Psychological consequences of awareness and their treatment. Best Pract Res Clin Anaesthesiol. 2007;21(3):357-67. 